\title{
Repercusiones de jugar sin público en la ventaja local, las decisiones arbitrales y en los componentes del juego
}

\section{Repercussions of playing without an audience on home advantage, refereeing decisions and game components}

\section{Repercussões de jogar sem público no factor casa, decisões da arbitragem e componentes do jogo}

\author{
Jiménez-Sánchez, Á ${ }^{1}$, Lavín, J. M. ${ }^{2}$, Endara, D. F. ${ }^{3}$ \\ ${ }^{1}$ Universidad de Salamanca, España; ${ }^{2}$ Centro Universitario CESINE, España; ${ }^{3}$ FLACSO, Ecuador.
}

\section{RESUMEN}

La facilitación social determina que la presencia de espectadores en los eventos deportivos es un factor favorable a la hora de jugar en casa. El objetivo de esta investigación es analizar la diferencia de puntos y goles en función de la presencia o ausencia de multitud, así como estudiar los posibles cambios en las decisiones arbitrales y en el estilo de juego tanto para los equipos locales como para los visitantes ante esta nueva situación generada por la pandemia del COVID-19. La muestra está conformada por los partidos jugados en la temporada 2019-2020 correspondientes a ocho ligas de fútbol europeo: Alemania, España, Italia, Inglaterra y Austria. Los resultados muestran que no hay diferencias significativas entre jugar con público o sin él, excepto en la primera categoría alemana y española. Sin embargo, se observa un aumento en los componentes defensivos y una reducción en los ofensivos en los equipos que juegan en casa sin espectadores. Como consecuencia, también hay cambios en las decisiones de los árbitros quienes asignaron menos faltas y tarjetas para los visitantes, excepto en ambas categorías alemanas y en la austriaca. Estos hallazgos no respaldan de manera generalizada la teoría de la facilitación social, pero sí su relación con la agresividad en los jugadores locales e incluso con las decisiones arbitrales. Las posibles implicaciones conducen especialmente a la preparación psicológica y entrenamiento táctico tanto de jugadores como de árbitros para afrontar encuentros sin público en los estadios.

Palabras clave: facilitación social, fútbol, árbitros, componentes del juego, espectadores.

\section{ABSTRACT}

Social facilitation determines that the presence of spectators at sporting events is a favorable factor when playing at home. The objective of this research is to analyze the difference in points and goals according to the 


\section{Jugar sin público y ventaja local}

presence or absence of a crowd, as well as to study the possible changes in refereeing decisions and in the style of playing for both local teams and visitors to this new situation generated by the COVID-19 pandemic. The sample is made up of the games played in the 2019th-2020th season corresponding to eight European football leagues: Germany, Spain, Italy, England and Austria. The results show that there are no significant differences between playing with or without the public, except in the first German and Spanish categories. However, an increase in defensive components and a reduction in offense are observed in teams that played at home without spectators. As a consequence, there are also changes in the referees' decisions who called fewer fouls and cards for the visitors, except in both the German and Austrian categories. These findings do not support the theory of social facilitation in a generalized way, but they do support its relationship with aggressiveness in local players and even with referee's decisions. The possible implications lead especially to the psychological preparation and tactical training of both players and referees to face matches without an audience in the stadiums.

Keywords: social facilitation, football, referees, game components, spectators.

\section{RESUMO}

A facilitação social determina que a presença de espectadores em eventos esportivos seja um fator favorável para jogar em casa. O objetivo desta pesquisa é analisar a diferença de pontos e gols em função da presença ou ausência de torcida, bem como estudar as possíveis mudanças nas decisões de arbitragem e no estilo de jogo das equipes locais e dos visitantes deste. nova situação gerada pela pandemia COVID-19. A amostra é composta pelos jogos disputados na temporada 2019-2020 correspondentes a oito ligas europeias de futebol: Alemanha, Espanha, Itália, Inglaterra e Áustria. Os resultados mostram que não há diferenças significativas entre jogar com ou sem público, exceto nas primeiras categorias alemã e espanhola. Porém, verifica-se um aumento dos componentes defensivos e uma redução do ataque em equipas que jogam em casa sem espectadores. Como consequência, há também mudanças nas decisões dos árbitros que atribuíram menos faltas e cartões aos visitantes, exceto nas categorias alemã e austríaca. Esses achados não apóiam a teoria da facilitação social de forma generalizada, mas apóiam sua relação com a agressividade dos jogadores locais e até mesmo com as decisões do árbitro. As possíveis implicações levam principalmente à preparação psicológica e ao treinamento tático de jogadores e árbitros para enfrentar jogos sem público nos estádios.

Palavras chave: facilitação social, futebol, árbitros, componentes do jogo, espectadores.

\section{INTRODUCCIÓN}

En varios contextos deportivos, los equipos rinden mejor cuando juegan en terreno local, es lo que comúnmente se conoce como "factor campo", ventaja de jugar como local o "ventaja en el hogar" (Home Advantage (HA)) (Neave y Wolfson, 2003). Esta superioridad está bien documentada en una amplia gama de deportes de equipo, incluido el fútbol (también llamado soccer en algunos países) (Goumas, 2014a; Page y Page, 2007; Pollard, 2008; Pollard y Gómez, 2014).

Algunas investigaciones relevantes sobre $\mathrm{HA}$ en el ámbito futbolístico muestran como este fenómeno se ha dado en España (Lago-Peñas y Lago-Ballesteros, 2011; Sánchez et al., 2009; Tucker, Mellalieu, James y Taylor, 2005), en la Premier League inglesa y en otras ligas europeas importantes (González-García y
Martínez-Martínez, 2019; Goumas, 2014b). También se observa por continentes (Pollard, 2007), durante décadas en algunas de las principales ligas de Europa (Pollard y Gómez, 2009; Rodríguez-Quijada, 2015; Saavedra-García, Gutiérrez-Aguilar, FernándezRomero y Sa-Marques, 2015), e incluso en algunos campeonatos femeninos europeos, aunque se ha observado un índice menor de victorias locales en comparación con los hombres (Pollard y Gómez, 2012). Así, aunque existan diferencias entre género, país, calidad de los equipos, década o deporte, los datos investigados corroboran de manera generalizada y sólidamente la relación entre la ubicación y el rendimiento de los equipos.

Por ello, una de las principales líneas de estudio son las causas que afectan a esa superioridad. Son cuatro las que generalmente se establecen: desplazamientos, 


\section{Jiménez-Sánchez et al.}

público, familiaridad y/o aprendizaje con el entorno y factores relacionados con las reglas (Courneya y Carron, 1992; García-Calvo et al., 2011; Jacklin, 2005; Loughead, Carron, Bray y Kim, 2003). Pero también existen otros elementos de gran interés como son los factores psicológicos, las decisiones de los expertos, la identidad del equipo o la territorialidad (García-Calvo et al., 2011; Gómez-Bantel, 2015; Hernández-Bonilla, 2019; Simonenkova y Sopov, 2020).

El planteamiento establecido es que estas variables afectarían cada una en mayor o menor medida en la HA a través de cambios en otras variables en función de que el equipo sea local o visitante. Así, las victorias en casa se explicarían por un incremento en las acciones ofensivas, ritmo de juego y en ocasiones también por la agresividad funcional en el equipo local (aumento de la activación fisiológica y la concentración), hipótesis argumentadas tanto por el análisis minucioso de los elementos del juego (McGuire, Courneya, Widmeyer y Carron, 1992) como incluso por los niveles de ciertas hormonas como el cortisol o la testosterona (Carolina-Paludo et al., 2020; Fothergill, Wolfson y Neave, 2017; Furley, Schweizer y Memmert, 2018; Neave y Wolfson, 2003), a la vez que el comportamiento táctico resulta ser más ofensivo para los equipos que juegan en casa respecto a la selección de un estilo más defensivo por parte de los visitantes (Glamser, 1990; Schwartz y Barsky, 1977; Seçkin, 2006), determinado especialmente por la precisión de tiro para las acciones ofensivas y por el número de tiros concedidos al defender (Brito-de Souza et al., 2019). En definitiva, lo que se plantea es que estas diferencias en el rendimiento estarían causadas por numerosas variables contextuales que beneficiarían a los equipos locales en detrimento de los visitantes (Inan, 2020) y donde la afición del estadio sería uno de los principales factores por el que se inclinan estos autores mencionados para poder explicar estas características de la ventaja local dada por la multitud o crowd advantage (CA) (Nevill, Balmer y Williams, 1999).

Las teorías sobre esta influencia del público se remontan a los orígenes de la psicología social. Uno de los primeros investigadores en mencionarlo es Triplett en 1898 y sus factores dinamogénicos al demostrar que la presencia de otros competidores aumentaba el arousal y mejoraba el rendimiento de los ciclistas profesionales.

Posteriormente, Allport (1924) denominaría "facilitación social" al efecto que ejerce la presencia de otras personas sobre el rendimiento de un individuo. A este concepto, Zajonc (1965) añadiría que el rendimiento influenciado por el público (activación aumentada) variaría según la experiencia del ejecutor, mejor cuanto más cualificado y peor cuanto menos experticia tiene este. Aplicado al deporte, cuando se produce un aumento de la presión del público hacia los deportistas (ya sea positiva o negativa), aquellos jugadores que poseen mayor experiencia y mayor control en estas situaciones tendrán más y mejor rendimiento, sin embargo, los jugadores más noveles serán más propensos a cometer un mayor número de errores (Cox, 2008; OlmedillaZafra, 2012).

Cox además aclara que la influencia no solo beneficia al equipo local, sino que además perjudica al equipo rival. Así, a mayor número de espectadores se puede percibir un aumento de las victorias en casa al tiempo que la hostilidad de la afición puede desorientar al rival dificultando que realice su juego. Por otro lado, la presión del público puede ser perjudicial para el equipo local, ya sea por las altas expectativas y presión del público hacia los jugadores para que ganen y jueguen bien o por un excesivo incremento en la activación debido a un público que grita y anima en exceso, estresando y desconcentrando al equipo animado (Baumeister y Steinhilber, 1984; Butler y Baumeister, 1998; Wright, Voyer, Wright y Roney, 1995).

Aunque la mayoría de las disciplinas deportivas de equipos corrijan este sesgo mediante partidos de ida y vuelta o encuentros en terreno neutro, el efecto del público variará en cada partido tanto en cantidad como en calidad hacia uno u otro equipo (Guschwan, 2015), provocando pequeñas diferencias entre ellos y su HA motivada por la afición.

Nevill, Newell y Gale (1996) comprobaron que la ventaja local estaba asociada con la media de asistencia al estadio, mientras que estudios como los de Dowie (1982) o Pollard (2008) no encontraron diferencias a favor del equipo de casa respecto a la cantidad de espectadores absoluta y su densidad. 


\section{Jugar sin público y ventaja local}

Finalmente, el público no solo podría influir en los jugadores, sino también en las decisiones de los árbitros (Picazo-Tadeo, González-Gómez y Guardiola, 2017). Nevill y sus colaboradores (1999) señalaron que una presencia de espectadores abundante y la generación de ruido pueden provocar un desequilibrio en las decisiones de los árbitros a favor del equipo local. Lehman y Reifman (1987) demostraron que los jugadores "estrella" eran sancionados en menor medida en casa que fuera. Y respecto a la relación entre la ubicación y las decisiones de expertos, se han confirmado desequilibrios arbitrales a favor de los equipos locales (Nevill et al., 1996).

La pandemia mundial del COVID-19 ha provocado que muchos espectáculos deportivos se jueguen sin público, una medida que ha afectado a las principales ligas de fútbol. Esta coyuntura permite un escenario natural con el que comprobar la influencia de la afición (antes-con espectadores / después-sin ellos), situación única hasta el momento que resolvería la validez externa echada en falta en este tipo de estudios (Jiménez-Sánchez y Lavín, 2020; Myers, 2014).

Por tanto, el objetivo de esta investigación es analizar las posibles diferencias en la HA de estas ligas y clubes dependiendo de la presencia o ausencia del público. Siguiendo la línea de la facilitación social (Zajonc, 1965), la principal hipótesis es que la HA sería más baja (menos puntos y goles a favor) sin la presencia o el apoyo de su afición local en el estadio y que los visitantes se beneficiarían de ello al no tener a la mayoría de espectadores en su contra.

Profundizando en este aspecto, se espera obtener un estilo de juego más defensivo o conservador en los equipos locales ante la ausencia de sus fans y un juego de tipo más ofensivo en los visitantes al no tener al público en contra.

Por último, se analizará esta posible influencia en la decisión de los árbitros. En el caso de que estos sí estuvieran afectados por la presión del público local, se esperaría que la ausencia de este repercutiera en un mayor número de amonestaciones y tarjetas en el equipo local y viceversa.

El motivo de analizar pormenorizadamente estos factores a la vez (componentes del juego y decisiones arbitrales) es la interrelación de ambos, es decir, que un descenso en el número de faltas o tarjetas al jugar sin público en los equipos visitantes puede deberse tanto a que los árbitros no tienen la presión de la afición en contra, como a que el equipo local juega menos ofensivamente, lo que conlleva esta disminución de faltas y tarjetas en los visitantes. Por tanto, al analizar estos dos aspectos conjuntamente se podrá esclarecer si los posibles cambios al jugar sin espectadores se deben a decisiones arbitrales o a cambios en el estilo de juego o sus componentes.

Estos resultados podrían permitir esclarecer si la HA está íntimamente ligada a la afición, o por otra parte, si la variable público actúa independientemente del factor casa.

\section{MATERIAL Y MÉTODOS}

\section{Diseño de investigación}

Investigación empírica con estrategia no manipulativa (asignación de forma natural), cuasi experimental con un diseño longitudinal de series temporales interrumpidas con medidas pretest y postest (con y sin espectadores) para cada uno de los ocho campeonatos (Ato, López, y Benavente, 2013).

\section{Muestra}

Se analizaron ocho ligas europeas cuyos partidos finales de la temporada 2019-2020 se jugaron sin público: La Bundesliga alemana de primera y segunda división, la Bundesliga Austriaca, la Liga Santander y Liga Smartbank (primera y segunda división del fútbol profesional español), la italiana también en ambas categorías (Serie A y B) y la inglesa Premier League.

Al ser la misma temporada y no otra nueva, cada liga siguió conformada por los mismos equipos, sin cambiar de plantilla y sus objetivos siguieron siendo los mismos que cuando dejaron de jugar con público. Esto ocurrió a principios de marzo de 2020, con un parón de dos meses aproximadamente y reanudándose a finales de mayo y principios de junio en estos países (con algunas excepciones).

De entre las diversas ligas en esta situación, se seleccionaron aquellas con un alto porcentaje de partidos jugados a puerta cerrada para así disponer de una muestra amplia a nivel general (más de 9 partidos cada equipo en esta situación) y que así también permita estudiar las diferencias entre equipos y el 


\section{Jiménez-Sánchez et al.}

factor campo (4 o 5 partidos en casa o fuera). A su vez, se toman en consideración clubes con diversos niveles y de diversas nacionalidades para poder comparar las posibles similitudes y diferencias (Tabla 1 y 2 ).

Las unidades de análisis son todos los partidos jugados por cada equipo. Así, si por ejemplo la Bundesliga alemana de primera división tuvo 306 encuentros, resultan en total 612 casos (18 equipos jugaron 34 partidos), de los cuales una mitad se jugó en casa y la otra, fuera (17 en cada campo). Es decir, aunque el total de encuentros sean 306, el resultado por cada uno de ellos es diferente para uno u otro equipo (local o visitante), por eso las unidades de análisis son los resultados por cada equipo (18 por 34) y no la mitad, aunque esta corresponda a la totalidad de partidos jugados en esa liga (306 en casa para unos, pero también 306 para los foráneos).

\section{Procedimiento}

Para el estudio se creó una tabla con cada competición que contenía datos técnicos como el nombre de la liga, el equipo, la jornada y la fecha; variables dependientes, entre las que se tuvieron en cuenta los goles a favor y en contra y los puntos ( 3 ganado / 1 empatado / 0 perdido) y el puesto en la clasificación; variables independientes, en donde se incluyó el antes con público y el después sin público; y el campo (casa
I fuera). Además, se añadieron otras variables relacionadas con aspectos arbitrales como las faltas y las tarjetas, teniendo en cuenta dentro de estas las amarillas, las rojas y el sumatorio de ambas (las rojas valían como dos amonestaciones). Asimismo, se contó con otros aspectos relacionados con los componentes del juego tanto ofensivo como defensivo: posesión $(\%)$, tiros totales (suma de tiros a puerta, fuera y disparos bloqueados), saques de esquina, ocasiones claras y entradas (acciones realizadas para quitar el balón al adversario). No se midieron los pases por considerarse acciones ambiguas, pues estos pueden ejecutarse tanto en una mitad del campo como en otra, por lo que no serían buenos indicadores para discriminar entre estilos de juego ofensivo o defensivo. Tampoco se incluyeron los regates por el mismo motivo, además de que tras un análisis preliminar no se encontró ninguna relación significativa entre los regates y el objeto de estudio.

Por último, se valoró también el presupuesto de cada equipo para comprobar si las posibles diferencias encontradas se dan más en determinados equipos respecto a su calidad (posición en la tabla), la cual, como se verá a continuación, está muy relacionada con la economía de cada club (Tabla 3 ).

A continuación se muestran los descriptivos obtenidos.

Tabla 1

Descriptivos. Ligas alemanas y españolas

\begin{tabular}{lcccc}
\hline $\begin{array}{l}\text { Descriptivos } \\
\text { (Sumatorios, medias, d.t., \%) }\end{array}$ & $\begin{array}{c}\text { Bundesliga } \\
\text { Alemana 1 }\end{array}$ & $\begin{array}{c}\text { Bundesliga } \\
\text { Alemana 2 }\end{array}$ & $\begin{array}{c}\text { LaLiga } \\
\text { España 1 }\end{array}$ & $\begin{array}{c}\text { LaLiga } \\
\text { España 2 }\end{array}$ \\
\hline Equipos & 18 & 18 & 20 & 22 \\
Partidos total & 612 & 612 & 760 & 922 \\
Partidos Antes (con público) & $446(72,9 \%)$ & $450(73,6 \%)$ & $538(70,8 \%)$ & $680(73,7 \%)$ \\
Partidos después (sin público) & $166(27,1 \%)$ & $162(26,4 \%)$ & $222(29,2 \%)$ & $242(26,3 \%)$ \\
Jornadas & 34 & 34 & 38 & 42 \\
Puntos (d.t.) & $1,39(1,3)$ & $1,34(1,2)$ & $1,36(1,2)$ & $1,33(1,2)$ \\
Goles (d.t.) & $1,6(1,3)$ & $1,4(1,2)$ & $1,2(1,1)$ & $1,1(1)$ \\
Presupuesto en millones de Euros (d.t.) & $236(204)$ & $18(12)$ & $258(268)$ & $14(10)$ \\
Tiros totales (d.t.) & $13,2(5)$ & $13,5(4,7)$ & $11,3(4,5)$ & $11,2(4,3)$ \\
Posesión en casa (d.t.) & $51,4 \%(12)$ & $50,8 \%(11)$ & $50,9 \%(11)$ & $50,9 \%(9)$ \\
Posesión fuera (d.t.) & $48,6 \%(12)$ & $49,2 \%(11)$ & $49,1 \%(11)$ & $49,1 \%(9)$
\end{tabular}




\section{Jugar sin público y ventaja local}

\begin{tabular}{lcccc} 
Corners (d.t.) & $5(3)$ & $5,1(2,7)$ & $4,6(2,5)$ & $4,4(2,5)$ \\
Faltas (d.t.) & $12,1(4)$ & $13(4)$ & $13,7(4,1)$ & $15,7(4,2)$ \\
T. Amarillas (d.t.) & $2(1,3)$ & $2(1,3)$ & $2,5(1,5)$ & $2,7(1,4)$ \\
T. Rojas (d.t.) & $0,1(0,3)$ & $0,1(0,3)$ & $0,1(0,3)$ & $0,1(0,3)$ \\
Tarjetas totales (d.t.) & $2,1(1,5)$ & $2,2(1,5)$ & $2,7(1,7)$ & $3(1,6)$ \\
Ocasiones (d.t.) & $2,4(1,8)$ & $2,1(1,5)$ & $1,8(1,5)$ & $1,5(1,3)$ \\
Entradas (d.t.) & $16,3(5,1)$ & $15(5,1)$ & $15(4,5)$ & $14,6(4,6)$ \\
\hline
\end{tabular}

Tabla 2

Descriptivos. Ligas italianas, inglesa y austriaca

\begin{tabular}{lcccc}
\hline $\begin{array}{l}\text { Descriptivos } \\
\text { (Sumatorios, medias, d.t., \%) }\end{array}$ & $\begin{array}{c}\text { Serie A } \\
\text { Italia 1 }\end{array}$ & $\begin{array}{c}\text { Serie B } \\
\text { Italia 2 }\end{array}$ & $\begin{array}{c}\text { Premier League } \\
\text { Inglaterra }\end{array}$ & $\begin{array}{c}\text { Bundesliga } \\
\text { Austria }\end{array}$ \\
\hline Equipos & 20 & 20 & 20 & 12 \\
Partidos total & 760 & 760 & 760 & 384 \\
Partidos Antes (con público) & $499(65,7 \%)$ & $528(69,5 \%)$ & $576(75,8)$ & $264(68,8 \%)$ \\
Partidos después (sin público) & $261(34,3 \%)$ & $232(30,5 \%)$ & $184(24,2)$ & $120(31,2 \%)$ \\
Jornadas & 38 & 38 & 38 & $32(22+10)$ \\
Puntos (d.t.) & $1,39(1,3)$ & $1,37(1,3)$ & $1,38(1,3)$ & $1,38(1,3)$ \\
Goles (d.t.) & $1,5(1,2)$ & $1,2(1)$ & $1,3(1,2)$ & $1,6(1,5)$ \\
Presupuesto en millones de Euros (d.t.) & $229(172)$ & $15(5)$ & $415(287)$ & $20(25)$ \\
Tiros totales (d.t.) & $14,3(5,6)$ & $13,2(4,8)$ & $12,3(5,4)$ & $12,8(5,5)$ \\
Posesión en casa (d.t.) & $50,1 \%(10)$ & $49,8 \%(9)$ & $50,1 \%(12)$ & $50,8 \%(10)$ \\
Posesión fuera (d.t.) & $49,9 \%(10)$ & $50,2 \%(9)$ & $49,9 \%(12)$ & $49,2 \%(10)$ \\
Corners (d.t.) & $5,3(3)$ & $4,7(2,4)$ & $5,3(3)$ & $5,1(2,9)$ \\
Faltas (d.t.) & $13,9(4)$ & $16(4,3)$ & $10,7(3,6)$ & $15,2(4,2)$ \\
T. Amarillas (d.t.) & $2,5(1,3)$ & $2,6(1,3)$ & $1,7(1,2)$ & $2,2(1,3)$ \\
T. Rojas (d.t.) & $0,1(0,3)$ & $0,1(0,4)$ & $0,06(0,2)$ & $0,1(0,3)$ \\
Tarjetas totales (d.t.) & $2,9(1,7)$ & $2,8(1,6)$ & $1,8(1,3)$ & $2,5(1,6)$ \\
Ocasiones (d.t.) & $2(1,6)$ & $1,8(1,4)$ & $2(1,6)$ & $2,2(1,8)$ \\
Entradas (d.t.) & $14,5(4,5)$ & $14(4,4)$ & $16,3(5,2)$ & $15,7(5,1)$ \\
\hline & & & &
\end{tabular}

Gran parte de la información se extrajo de las siguientes fuentes:

- https://sofascore.com

- www.transfermarkt.es

Ambas páginas web son de reconocido prestigio y respondieron correctamente a la prueba de calidad del dato realizada con diez partidos aleatorios de cada liga.

Análisis estadístico
Una vez recopilada la información se trabajó con el programa estadístico SPSS (versión 23 para Windows). Las pruebas utilizadas fueron el Chicuadrado de Pearson, la $t$-Student, correlación de Pearson y el modelo lineal general univariado ( $\mathrm{p}<$ $0,05)$. Por último, cabe mencionar que en las variables estudiadas las pruebas de normalidad y de homocedasticidad dieron un valor de $p<0,05$ en casi todas las competiciones, lo que significa que no cumplían ambos supuestos. 


\section{Jiménez-Sánchez et al.}

\section{RESULTADOS}

Antes de presentar los análisis principales es necesario mencionar una serie de correlaciones fundamentales (Pearson, $\mathrm{p}<0,05$ ). Así, de manera generalizada y bidireccionalmente, un mejor puesto en la clasificación, más goles a favor y puntos se correlacionan con un mayor presupuesto del equipo, más posesión del balón, tiros, ocasiones y menos tarjetas por partido. Estos datos discrepan ligeramente en función de la liga analizada y es aconsejable conocerlas para entender posteriormente los resultados encontrados (ver Anexos).
A continuación se muestra la correlación de estos aspectos con los puntos (con público y sin él y tanto en casa como fuera) para tener una orientación aproximada del comportamiento de cada competición (Tabla 3), donde se observa que no todas responden de igual manera. Si bien el campo local se asociaría con más victorias y estas últimas con una mayor posesión del balón (Errekagorri-Elezkano, EcheazarraEscudero y Castellano-Paulis, 2020), se encuentra que la posesión sí lo hace significativamente con los puntos (con un par de excepciones) pero sin embargo, el factor campo solo correlaciona de manera significativa con la posesión (más en casa) en los casos de la liga germana de primera división y en la española tanto de primera como de segunda categoría.

Tabla 3

Correlación de Pearson y $p$ valor entre puntos y variables

\begin{tabular}{lcccccccc}
\hline Liga & Valor (Presupuesto) & Posesión & Tiros & Corners & Faltas & Ocasiones & Entradas & Tarjetas \\
\hline Alemania 1 & $0,313^{* *}$ & $0,159^{* *}$ & $0,164^{* *}$ & 0,002 & $-0,058$ & $0,454^{* *}$ & 0,025 & $-0,111^{* *}$ \\
$\mathrm{~N}=612$ & 0,000 & 0,000 & 0,000 & 0,967 & 0,149 & 0,000 & 0,530 & 0,006 \\
& & & & & & & \\
Alemania 2 & $0,107^{* *}$ & $-0,070$ & 0,073 & $-0,091^{*}$ & $-0,004$ & $0,359^{* *}$ & $-0,007$ & $-0,116^{* *}$ \\
$\mathrm{~N}=612$ & 0,008 & 0,086 & 0,070 & 0,024 & 0,931 & 0,000 & 0,866 & 0,004 \\
LaLiga1 & $0,259^{* *}$ & 0,043 & $0,150^{* *}$ & $-0,026$ & 0,021 & $0,424^{* *}$ & 0,007 & $-0,113^{* *}$ \\
$\mathrm{~N}=760$ & 0,000 & 0,231 & 0,000 & 0,482 & 0,564 & 0,000 & 0,853 & 0,002 \\
LaLiga2 & $0,104^{* *}$ & $-0,256^{* *}$ & 0,034 & $-0,092^{* *}$ & $0,115^{* *}$ & $0,391^{* *}$ & 0,032 & $-0,090^{* *}$ \\
$\mathrm{~N}=922$ & 0,002 & 0,000 & 0,307 & 0,005 & 0,000 & 0,000 & 0,335 & 0,006 \\
Serie A & $0,295^{* *}$ & $0,138^{* *}$ & $0,243^{* *}$ & 0,044 & 0,017 & $0,385^{* *}$ & 0,034 & $-0,061$ \\
$\mathrm{~N}=760$ & 0,000 & 0,000 & 0,000 & 0,221 & 0,640 & 0,000 & 0,346 & 0,091 \\
Serie B & 0,011 & $-0,214^{* *}$ & $0,150^{* *}$ & $-0,014$ & $-0,034$ & $0,343^{* *}$ & 0,046 & $-0,064$ \\
$\mathrm{~N}=760$ & 0,758 & 0,000 & 0,000 & 0,701 & 0,344 & 0,000 & 0,205 & 0,078 \\
& $0,291^{* *}$ & $0,107^{* *}$ & $0,270^{* *}$ & $0,094^{* *}$ & 0,051 & $0,424^{* *}$ & 0,039 & $-0,077^{*}$ \\
Premier League & 0,000 & 0,003 & 0,000 & 0,009 & 0,159 & 0,000 & 0,280 & 0,033 \\
$\mathrm{~N}=760$ & $0,263^{* *}$ & $0,159^{* *}$ & $0,330^{* *}$ & $0,176^{* *}$ & 0,005 & $0,471^{* *}$ & $-0,011$ & $-0,240^{* *}$ \\
Austria & 0,000 & 0,002 & 0,000 & 0,001 & 0,920 & 0,000 & 0,835 & 0,000 \\
$\mathrm{~N}=384$ & & & & & & & &
\end{tabular}

Notas: $* p<, 05 . * * p<, 01$.

$\mathrm{p}=0,013)$ y en la primera categoría de la española $(F$ $=5,463 ; \mathrm{p}=0,020)$, ambas con menos puntos en casa y más fuera al jugar sin espectadores. Sin embargo, se puede hablar de una tendencia hacia estos últimos resultados también en los casos de la liga austriaca y la Serie B italiana $(\mathrm{p}=0,100$ y $\mathrm{p}=0,135$ respectivamente).

Los análisis muestran que apenas han variado los puntos por partido al jugar sin afición. El modelo lineal general entre los puntos (variable dependiente), el campo (casa o fuera) y el público (con o sin), no revela diferencias significativas ( $p>0,05)$, excepto en la Bundesliga alemana de primera división $(F=6,172$; 


\section{Jugar sin público y ventaja local}

De manera similar ocurre con los goles a favor y en contra en cada partido, donde no hay diferencias significativas en función de jugar con o sin público y el terreno de juego, excepto en la liga austriaca con menos goles a favor en casa al estar sin espectadores que con ellos $(t=2,074 ; \mathrm{p}=0,040)$ y también casi significativamente en la liga de primera categoría alemana y española $(t=1,736 ; \mathrm{p}=0,084$ y $t=1,838$; $\mathrm{p}=0,067$ respectivamente) .

No hay diferencias con el número de goles en contra, lo que da a entender que la reducción de puntos en casa ante la ausencia de espectadores se debe especialmente al menor número de goles por parte del equipo local y no tanto de los rivales y, aunque los tantos en contra sean casi los mismos, este hecho es lo suficientemente decisivo para que los clubes que juegan fuera puedan beneficiarse en detrimento de los locales. En la Tabla 4 y 5 se muestran los datos correspondientes teniendo en cuenta que una $t$ de Student positiva significa que al jugar sin público se puntuó menos en la variable contrastada y una t de valor negativo que sin espectadores se aumentó la cifra en dicha variable.

Tabla 4

$t$ de Student ( $p$ valor) entre público y otras variables según el campo de juego. Competiciones alemanas y españolas

\begin{tabular}{lccccc}
\hline $\begin{array}{l}\text { Público (con y sin) } \\
\text { y Variables }\end{array}$ & Campo & Bundesliga 1 & Bundesliga 2 & LaLiga 1 & LaLiga 2 \\
\hline Posesión & Casa & $\mathrm{t}=0,480(0,631)$ & $\mathrm{t}=-0,290(0,772)$ & $\mathrm{t}=-0,657(0,511)$ & $\mathrm{t}=1,042(0,298)$ \\
& Fuera & $\mathrm{t}=-0,647(0,518)$ & $\mathrm{t}=0,035(0,972)$ & $\mathrm{t}=0,521(0,603)$ & $\mathrm{t}=-0,964(0,335)$ \\
Tiros totales & Casa & $\mathrm{t}=2,085(\mathbf{0 , 0 3 8})$ & $\mathrm{t}=3,899(\mathbf{0 , 0 0 0})$ & $\mathrm{t}=3,407(\mathbf{0 , 0 0 1})$ & $\mathrm{t}=4,335(\mathbf{0 , 0 0 0})$ \\
& Fuera & $\mathrm{t}=0,777(0,438)$ & $\mathrm{t}=0,470(0,638)$ & $\mathrm{t}=-0,662(0,508)$ & $\mathrm{t}=1,096(0,273)$ \\
Corners & Casa & $\mathrm{t}=1,421(0,157)$ & $\mathrm{t}=1,171(0,243)$ & $\mathrm{t}=3,150(\mathbf{0 , 0 0 2})$ & $\mathrm{t}=2,962(\mathbf{0 , 0 0 3})$ \\
& Fuera & $\mathrm{t}=0,150(0,881)$ & $\mathrm{t}=0,555(0,580)$ & $\mathrm{t}=-0,497(0,619)$ & $\mathrm{t}=0,189(0,850)$ \\
Faltas & Casa & $\mathrm{t}=-2,269(\mathbf{0 , 0 2 4})$ & $\mathrm{t}=-1,833(0,068)$ & $\mathrm{t}=0,365(0,715)$ & $\mathrm{t}=-0,045(0,964)$ \\
& Fuera & $\mathrm{t}=-0,503(0,615)$ & $\mathrm{t}=1,042(0,298)$ & $\mathrm{t}=1,607(0,109)$ & $\mathrm{t}=1,023(0,307)$ \\
T. Amarillas & Casa & $\mathrm{t}=-1,909(0,059)$ & $\mathrm{t}=-2,699(\mathbf{0 , 0 0 7})$ & $\mathrm{t}=0,319(0,750)$ & $\mathrm{t}=-0,806(0,421)$ \\
T. Rojas & Fuera & $\mathrm{t}=0,875(0,382)$ & $\mathrm{t}=1,410(0,160)$ & $\mathrm{t}=3,366(\mathbf{0 , 0 0 1})$ & $\mathrm{t}=2,251(\mathbf{0 , 0 2 5})$ \\
& Casa & $\mathrm{t}=-0,491(0,624)$ & $\mathrm{t}=2,011(\mathbf{0 , 0 4 6})$ & $\mathrm{t}=-1,098(0,274)$ & $\mathrm{t}=-0,029(0,997)$ \\
Tarjetas totales & Fuera & $\mathrm{t}=0,853(0,395)$ & $\mathrm{t}=-0,189(0,850)$ & $\mathrm{t}=0,669(0,504)$ & $\mathrm{t}=0,211(0,833)$ \\
& Casa & $\mathrm{t}=-1,828(0,070)$ & $\mathrm{t}=-1,649(0,100)$ & $\mathrm{t}=-0,302(0,763)$ & $\mathrm{t}=-0,881(0,379)$ \\
Ocasiones & Fuera & $\mathrm{t}=1,141(0,255)$ & $\mathrm{t}=1,025(0,306)$ & $\mathrm{t}=3,490(\mathbf{0 , 0 0 1})$ & $\mathrm{t}=1,399(0,162)$ \\
& Casa & $\mathrm{t}=1,512(0,132)$ & $\mathrm{t}=0,196(0,845)$ & $\mathrm{t}=3,769(\mathbf{0 , 0 0 0})$ & $\mathrm{t}=0,183(0,855)$ \\
Entradas & Fuera & $\mathrm{t}=-0,299(0,765)$ & $\mathrm{t}=-1,330(0,184)$ & $\mathrm{t}=0,518(0,605)$ & $\mathrm{t}=-0,641(0,522)$ \\
& Casa & $\mathrm{t}=1,063(0,289)$ & $\mathrm{t}=2,826(\mathbf{0 , 0 0 5})$ & $\mathrm{t}=1,722(0,086)$ & $\mathrm{t}=2,037(\mathbf{0 , 0 4 2})$ \\
& Fuera & $\mathrm{t}=2,947(\mathbf{0 , 0 0 3})$ & $\mathrm{t}=3,328(\mathbf{0 , 0 0 1})$ & $\mathrm{t}=1,513(0,131)$ & $\mathrm{t}=2,914(\mathbf{0 , 0 0 4})$ \\
\hline & & & & &
\end{tabular}

Tabla 5

$t$ de Student ( $p$ valor) entre público y otras variables según el campo de juego. Competiciones italianas, inglesa y austriaca

\begin{tabular}{lccccc}
\hline $\begin{array}{l}\text { Público (con y sin) } \\
\text { y Variables }\end{array}$ & Campo & Serie A & Serie B & Premier League & Austria \\
\hline Posesión & Casa & $\mathrm{t}=0,386(0,700)$ & $\mathrm{t}=0,646(0,519)$ & $\mathrm{t}=0,901(0,368)$ & $\mathrm{t}=0,913(0,362)$
\end{tabular}




\section{Jiménez-Sánchez et al.}

\begin{tabular}{lcccccc}
\multirow{4}{*}{ Tiros totales } & Fuera & $\mathrm{t}=-0,259(0,796)$ & $\mathrm{t}=-0,633(0,527)$ & $\mathrm{t}=-0,996(0,320)$ & $\mathrm{t}=-0,939(0,349)$ \\
Corners & Casa & $\mathrm{t}=2,697(\mathbf{0 , 0 0 7})$ & $\mathrm{t}=1,441(0,151)$ & $\mathrm{t}=2,945(\mathbf{0 , 0 0 3})$ & $\mathrm{t}=2,851(\mathbf{0 , 0 0 5})$ \\
& Fuera & $\mathrm{t}=-0,925(0,355)$ & $\mathrm{t}=-0,714(0,476)$ & $\mathrm{t}=0,054(0,957)$ & $\mathrm{t}=-0,362(0,718)$ \\
Faltas & Casa & $\mathrm{t}=3,021(\mathbf{0 , 0 0 3})$ & $\mathrm{t}=2,492(\mathbf{0 , 0 1 3})$ & $\mathrm{t}=2,010(\mathbf{0 , 0 4 5})$ & $\mathrm{t}=2,160(\mathbf{0 , 0 3 2})$ \\
& Fuera & $\mathrm{t}=-1,472(0,142)$ & $\mathrm{t}=-1,706(0,089)$ & $\mathrm{t}=-0,150(0,881)$ & $\mathrm{t}=-0,506(0,614)$ \\
T. Amarillas & Casa & $\mathrm{t}=0,916(0,360)$ & $\mathrm{t}=0,666(0,506)$ & $\mathrm{t}=-1,700(0,090)$ & $\mathrm{t}=-0,741(0,460)$ \\
& Fuera & $\mathrm{t}=3,020(\mathbf{0 , 0 0 3})$ & $\mathrm{t}=0,593(0,554)$ & $\mathrm{t}=-0,877(0,381)$ & $\mathrm{t}=1,456(0,147)$ \\
T. Rojas & Casa & $\mathrm{t}=2,478(\mathbf{0 , 0 1 4})$ & $\mathrm{t}=0,558(0,577)$ & $\mathrm{t}=1,696(0,091)$ & $\mathrm{t}=-1,775(0,078)$ \\
& Fuera & $\mathrm{t}=5,961(\mathbf{0 , 0 0 0})$ & $\mathrm{t}=2,341(\mathbf{0 , 0 2 0})$ & $\mathrm{t}=3,312(\mathbf{0 , 0 0 1})$ & $\mathrm{t}=1,380(0,169)$ \\
Tarjetas totales & Casa & $\mathrm{t}=1,101(0,272)$ & $\mathrm{t}=-0,945(0,336)$ & $\mathrm{t}=0,159(0,873)$ & $\mathrm{t}=-1,731(0,087)$ \\
& Fuera & $\mathrm{t}=2,068(\mathbf{0 , 0 3 9})$ & $\mathrm{t}=1,576(0,116)$ & $\mathrm{t}=0,044(0,965)$ & $\mathrm{t}=0,298(0,766)$ \\
Ocasiones & Casa & $\mathrm{t}=2,012(\mathbf{0 , 0 4 5})$ & $\mathrm{t}=-0,455(0,650)$ & $\mathrm{t}=1,368(0,173)$ & $\mathrm{t}=-2,194(\mathbf{0 , 0 2 9})$ \\
& Fuera & $\mathrm{t}=5,647(\mathbf{0 , 0 0 0})$ & $\mathrm{t}=2,765(\mathbf{0 , 0 0 6})$ & $\mathrm{t}=3,031(\mathbf{0 , 0 0 3})$ & $\mathrm{t}=1,092(0,276)$ \\
Entradas & Casa & $\mathrm{t}=0,132(0,895)$ & $\mathrm{t}=1,360(0,175)$ & $\mathrm{t}=0,936(0,350)$ & $\mathrm{t}=1,549(0,123)$ \\
& Fuera & $\mathrm{t}=0,474(0,636)$ & $\mathrm{t}=1,052(0,293)$ & $\mathrm{t}=1,599(0,111)$ & $\mathrm{t}=1,355(0,177)$ \\
& Casa & $\mathrm{t}=3,978(\mathbf{0 , 0 0 0})$ & $\mathrm{t}=-0,107(0,915)$ & $\mathrm{t}=3,679(\mathbf{0 , 0 0 0})$ & $\mathrm{t}=1,306(0,193)$ \\
& Fuera & $\mathrm{t}=3,608(\mathbf{0 , 0 0 0})$ & $\mathrm{t}=1,432(0,153)$ & $\mathrm{t}=3,249(\mathbf{0 , 0 0 1})$ & $\mathrm{t}=1,491(0,138)$ \\
\hline
\end{tabular}

Así, en ninguna liga se encuentran diferencias significativas en el porcentaje de posesión del balón en función del público, por lo que este no influiría en la tendencia observada anteriormente en ciertos campeonatos de hacer menos puntos y goles en casa sin espectadores y más fuera.

La reducción de tiros y de saques de esquina por parte del equipo local al jugar sin público y de acciones realizadas para quitar el balón al adversario (entradas) hechas por los visitantes denotarían una disminución del juego ofensivo para los locales y sin espectadores. Excepto la liga italiana de segunda división y la primera alemana, la tendencia también en los clubes que juegan en casa es a hacer menos entradas sin que la posesión, tiros, saques de esquina y ocasiones de gol varíen en los visitantes, lo que podría indicar una bajada en la agresividad por parte de los locales sin cambios en los componentes del juego de los foráneos.

La reducción de faltas o tarjetas en los visitantes al estar sin público en la mayoría de competiciones podría llevar a pensar que es debido a la teoría comentada sobre la presión en los árbitros del público y que en ausencia de esta presión los jueces tenderían a no perjudicar tanto a los que juegan fuera. Sin embargo, la teoría también predeciría un aumento en estos indicadores en los equipos locales, pues los árbitros no estarían presionados por estos aficionados locales, pero estos resultados no se han encontrado en los casos mencionados, sino que solo ha afectado a los visitantes, e incluso en la liga italiana de primera los que jugaron en casa también disminuyeron en número de tarjetas. Por tanto, la explicación más razonable a la reducción de faltas y amonestaciones en los visitantes es que se deba a un estilo de juego menos ofensivo al jugar sin espectadores por parte de los locales, si bien se observan algunas excepciones contradictorias que podrían explicarse por las decisiones arbitrales ante la ausencia de la afición local.

Sobre si estos cambios se dan más en unos equipos que en otros, el modelo lineal general univariado para los partidos en casa o fuera y, teniendo en consideración por un lado el presupuesto y el factor público y por otra parte los indicadores estudiados como variables dependientes, revela que no existen diferencias ( $p$ > 0,05 ) en ninguna liga respecto a los puntos obtenidos tanto en casa como fuera, excepto en la segunda categoría italiana, donde en campo local jugaron peor $\sin$ audiencia a mayor presupuesto de los clubes $(F=$ 


\section{Jugar sin público y ventaja local}

1,933; $p=0,013)$. Los resultados significativos en este ámbito se muestran a continuación, tomando siempre en consideración que las correlaciones expuestas también operan de manera inversa (dos colas).

-Alemania 1: En casa sin público, más tarjetas amarillas, especialmente en equipos de menor presupuesto $(F=1,981 ; \mathrm{p}=0,013)$. De igual forma con el número de tarjetas totales $(F=2,024 ; \mathrm{p}=$ $0,010)$. Fuera, más entradas a mayor presupuesto sin público $(F=2,053 ; \mathrm{p}=0,009)$.

-Alemania 2: Fuera, menos posesión en equipos grandes sin espectadores $(F=2,068 ; \mathrm{p}=0,009)$. También en esta situación menos tarjetas rojas en general, pero especialmente menos para clubes con menos presupuesto $(F=1,765 ; \mathrm{p}=0,032)$.

-España 2: En casa, más faltas realizadas por equipos con menor presupuesto sin público $(F=1,658 ; \mathrm{p}=$ 0,035). A su vez, menos entradas en equipos con menor presupuesto $(F=1,599 ; \mathrm{p}=0,046)$. Fuera y sin espectadores más faltas en clubes de menor presupuesto $(F=2,200 ; \mathrm{p}=0,002)$.

-Italia 1: Fuera, menos ocasiones a mayor presupuesto $(F=1,808 ; \mathrm{p}=0,021)$.

-Italia 2: En casa, menos tarjetas rojas en equipos con menor presupuesto al jugar sin espectadores $(F=$ 2,796; $\mathrm{p}=0,000)$. Fuera, menos corners a menor presupuesto sin público $(F=2,202 ; \mathrm{p}=0,009)$.

-Inglaterra: En casa, menos posesión a menor presupuesto sin afición $(F=1,647 ; \mathrm{p}=0,044)$. Fuera, menos posesión sin espectadores a menor presupuesto $(F=1,773 ; \mathrm{p}=0,025)$. También, más ocasiones a mayor presupuesto $(F=1,688 ; \mathrm{p}=0,036)$.

-Austria: En casa y sin público, menos posesión a más presupuesto $(F=2,566 ; \mathrm{p}=0,006)$. A su vez bajo la misma situación menos tarjetas rojas a mayor presupuesto $(F=4,021 ; \mathrm{p}=0,000)$.

\section{DISCUSIÓN Y CONCLUSIONES}

No es posible confirmar la hipótesis de que sin espectadores se obtienen menos puntos y goles en casa y más fuera, pero tampoco se puede afirmar en su totalidad que el público no influye en los jugadores según el terreno de juego. Los casos más aproximados son la primera división alemana, española (menos puntos y goles en campo local y más puntos en terreno contrario sin afición) y austriaca (menos goles en casa), sin embargo, no se encuentra un patrón aparente entre estas competiciones o entre las ligas donde no sucede lo mismo como para dar respuesta a por qué ocurre esto.

Ante estos resultados cabe preguntarse por qué muchos equipos siguen ganando más en casa que fuera sin la ausencia de público. Aparte de los argumentos mencionados en la introducción (familiaridad, factor identitario, cansancio por viajes, etc.), los datos hallados sobre los componentes del juego podrían ofrecer una pista aproximada al relacionarse con el tema de la agresividad y la territorialidad porque, aunque estos sean factores más de tipo evolutivo, sí parecen interactuar con lo social estudiado.

Las investigaciones sobre ello (Fothergill et al., 2017; Furley et al., 2018; Neave y Wolson, 2003; Pollard, 2007; Varca, 1980) muestran como los jugadores que juegan en casa tienen un incremento de la testosterona (mejor rendimiento) y al mismo tiempo genera una agresividad disfuncional en los rivales (peor juego y más faltas), lo que podría explicar la HA aun sin la presencia de espectadores. No obstante, habría que analizar si este sentido de territorialidad también se da en el público, de qué manera y en qué casos, pues entonces este podría ser un factor incidente en la HA. Así, los resultados de esta investigación han mostrado una tendencia a la reducción de goles en casa sin espectadores y la adaptación a un estilo de juego menos ofensivo, algo que podría estar provocado por esta disminución de la agresividad en los locales al tener menos público. También podría deberse a otros motivos como la baja forma y la acumulación de partidos durante esas últimas jornadas, sin embargo, parece que estas causas no explicarían los datos obtenidos, puesto que tanto la baja forma como la acumulación deberían afectar a todos los equipos por igual, mientras que los resultados se aplican especialmente a los equipos que juegan en casa y no tanto a los visitantes.

Por tanto, más que hablar de facilitación social, habría que tratarlo de presión social, ya que los asistentes al estadio, más que facilitar el juego a su equipo, lo que harían es presionar a sus deportistas para que sigan jugando a buen ritmo y no se acomoden o conformen con marcar el mínimo de goles para lograr la victoria. 


\section{Jiménez-Sánchez et al.}

Este planteamiento explicaría que no se encuentren diferencias significativas en los puntos y goles al jugar sin espectadores, pero sí un cambio hacia un estilo de juego más defensivo o conservador por parte de los locales, insuficiente como para inclinar la balanza hacia los visitantes, pero suficiente como para que los jugadores y sus entrenadores se replanteen el cambio táctico generado por la ausencia de los aficionados.

Respecto a la decisión de los jueces, en la mayoría de campeonatos hay una reducción en faltas y tarjetas por parte de los visitantes al jugar sin público, pero más que deberse a que los árbitros ya no tenían la presión del público local por sancionar a los rivales (que también podría ser), habría que achacarlo a la reducción de acciones ofensivas por parte de los que juegan en casa, lo que conlleva que los foráneos reduzcan la necesidad de hacer faltas y terminen con menos tarjetas. Por otra parte, en terreno amigo no se encuentra una aparente presión del público en las decisiones de los jueces, sino que a veces es todo lo contrario, con menos faltas y tarjetas en los locales y no al contrario tal y como plantearían los estudios sobre la influencia de espectadores en los árbitros (Nevill et al., 1999; Nevill et al., 1996; Unkelbach y Memmert, 2010). Sin embargo, en ambas categorías alemanas y en la austriaca sí ha habido un aumento de estos indicadores en los jugadores de casa y sin público, por lo que habría que seguir profundizando sobre ello para saber si los árbitros son más susceptibles de ser influenciados por los aficionados en ciertos campeonatos, o si bien existen otros motivos subyacentes que expliquen los resultados obtenidos.

Estos argumentos de agresividad y arbitraje también pueden servir para explicar los resultados encontrados en otras investigaciones sobre el género femenino tanto en el fútbol como en otros deportes (Arboix-Alió et al., 2020; Baena, Baena y del Pozo, 2009; Pollard y Gómez, 2012), con menor número de victorias y tantos en casa respecto a los hombres y con menos faltas para las jugadoras locales, pues si bien puede explicarse debido a la disminución de testosterona en las mujeres, también podría deberse a una menor asistencia de público en las ligas femeninas, lo que conllevaría una menor estimulación del arousal en las deportistas y, por tanto, un juego más defensivo o conservador que el observado en las grandes ligas analizadas masculinas. Por ello, queda pendiente analizar en profundidad si en el fútbol femenino se pitan más faltas jugando en casa debido a la menor presión de los árbitros por parte del escaso público asistente (Aranda-Malavés, Tudela-Desantes y Sanz-Ramírez, 2019) o si bien se hacen menos faltas debido a esta bajada de agresividad y ritmo de juego respecto a los hombres, sin descartar en cualquier caso que ambas opciones y otras más (culturales, fisiológicas, etc.) pueden intervenir simultáneamente.

Sobre ello, cabe mencionar que en un principio esta investigación quiso analizar las ligas femeninas para esclarecer este aspecto, pero lamentablemente no se encontraron datos suficientes ni tan precisos como en sus homólogas masculinas, por lo que se advierte de la dificultad de estudiar el fútbol femenino al mismo nivel expuesto en este artículo.

A su vez, se aconseja para futuras investigaciones profundizar en otros aspectos relacionados con el público como la implicación a la hora de animar a su equipo, la agresividad hacia los rivales, su intensidad, o la recepción según qué jugadores ante la presencia o ausencia de su afición. También, se recomienda ampliar la muestra en la medida de lo posible, pues el volumen de partidos por equipo analizados ( 9 o $10 \mathrm{sin}$ público y la mitad en casa aproximadamente) es relativamente bajo como para dar respuestas categóricas al comportamiento de cada uno ante esta nueva situación, aunque sí se considera suficiente como para hablar, no de clubes concretos, pero sí de grupos que reaccionan de manera diferente al jugar sin espectadores.

Por su parte, el presupuesto o valor de un equipo se muestra como una covariable que seguir estudiando con detenimiento. Dado que no existe una homogeneidad clara entre los países investigados, sino significaciones divergentes, se hace pertinente profundizar en aquellas características relacionadas con la partida presupuestaria de los clubes, como pudieran ser el aforo del estadio, el número de jugadores extranjeros, canteranos, u otros factores donde el público o su ausencia pudieran determinar la efectividad del juego.

En resumen, esta investigación muestra como la HA puede actuar de manera independiente de la denominada "ventaja por multitud" o crowd advantage (CA) (Nevill et al., 1999), es decir, que el posible beneficio de jugar en casa no tiene por qué depender solo de la afición asistente al estadio y que además, la 


\section{Jugar sin público y ventaja local}

influencia de esta varía según el campeonato, la categoría, el país y también afecta de forma diferente a los diversos componentes del juego de cada plantilla.

\section{APLICACIONES PRÁCTICAS}

Mientras la HA es compensada con los partidos de ida y vuelta, los posibles sesgos de la CA no son tan fáciles de evitar, actuando en beneficio de unos equipos y en detrimento de otros independientemente del factor campo. Si bien la HA no es algo que se diera en todas las ligas como una máxima futbolística, con la ausencia de público en ciertas competiciones, la reducción de victorias locales derivaría en un replanteamiento de la propia HA. En este sentido, no solo habría que preparar psicológica y tácticamente a los equipos (e incluso a los árbitros (Borrueco, Alcaraz, Ramis y Cruz, 2019)) para afrontar las consecuencias de un partido sin espectadores, sino también abarcar a otros sectores afectados como por ejemplo las apuestas deportivas, las cuales ya no deberían dar por hecho tan fácilmente que el resultado con mayor probabilidad es la victoria local.

\section{REFERENCIAS}

1. Allport, F. H. (1924). Social psychology. Boston: Houghton Mifflin.

2. Aranda-Malavés, R., Tudela-Desantes, A., y Sanz-Ramírez, E. (2019). Fútbol femenino: En la UEFA champions league femenina hay menos igualdad y se cometen más faltas que en la masculina. Revista internacional de deportes colectivos, $\quad 38, \quad 5-14$. http://www.asesdeco.com/images/pdf/38Aranda

3. Arboix-Alió, J., Buscà, B., Aguilera-Castells, J., Trabal-Taña, G., y Sánchez-López, M. J. (2020). Comparación de la ventaja de jugar en casa según género en la liga portuguesa de hockey sobre patines. Cuadernos de Psicología del Deporte, 20(1), 181-189. https://doi.org/10.6018/cpd.363041

4. Ato, M., López, J. J., y Benavente, A. (2013). Un sistema de clasificación de los diseños de investigación en psicología. Anales de Psicología, 29(3), 1038-1059. http://dx.doi.org/10.6018/analesps.29.3.178511
5. Baena, A., Baena, J., y del Pozo, B. (2009). Análisis de la frecuencia de faltas señaladas en función del género y el "home advantage" en categoría cadete. Cuadernos de Psicología del Deporte, https://revistas.um.es/cpd/article/view/85881

6. Baumeister, R. F., y Steinhilber, A. (1984). Paradoxical effects of supportive audiences on performance under pressure: The home advantage in sports championships. Journal of personality and psychology. 47(1), 85-93. https://doi.org/10.1037/0022-3514.47.1.85

7. Borrueco, M., Alcaraz, S., Ramis, Y., y Cruz, J. (2019). "No es solo pitar": diseño e implementación de un taller de formación para árbitros y jueces de deporte escolar. Revista de Psicología Aplicada al Deporte y al Ejercicio Físico, 4(2), 1-7. https://doi.org/10.5093/rpadef2019a11

8. Brito de Souza, D., López-Del Campo, R., Blanco-Pita, H., Resta, R., y Del Coso, J. (2019). An Extensive Comparative Analysis of Successful and Unsuccessful Football Teams in LaLiga. Front. Psychol., $\quad 10(2566), \quad 1-8$. https://doi.org/10.3389/fpsyg.2019.02566

9. Butler, J. L., y Baumeister, R. F. (1998). The trouble with friendly faces: Skilled performance with a supportive audience. Journal of personality and social psychology 75(5), 1213 - 1230. https://doi.org/10.1037//0022-3514.75.5.1213.

10. Carolina-Paludo, A., Nunes-Rabelo, F., MacielBatista, M., Rúbila-Maciel, I., PeikriszwiliTartaruga, M., y Simões, C. A. (2020). Game Location Effect on Pre-competition Cortisol Concentration and Anxiety State: A Case Study in a Futsal Team. Revista De Psicología Del Deporte, 29(1), 105-112. https://www.rpdonline.com/index.php/rpd/article/view/40

11. Courneya, K. S., y Carron, A. V. (1992). The home advantage in sport competitions: A literature review. Journal of Sport and Exercise Phychology, 14(1), 13-27. https://doi.org/10.1123/jsep.14.1.13 


\section{Jiménez-Sánchez et al.}

12. Cox, R. (2008). Psicología del deporte: conceptos y sus aplicaciones. Madrid: Médica Panamericana.

13. Dowie, J. (1982). Why Spain Should Win the World Cup. New Scientist, 94(10), 693-695. https://www.researchgate.net/profile/Jack_Dowie /publication/284600911_Why_Spain_should_wi n_the_World_Cup/links/5e81d906458515efa0b8 ed5e/Why-Spain-should-win-the-World-Cup.pdf

14. Errekagorri-Elezkano, I., Echeazarra-Escudero, I., y Castellano-Paulis, J. (2020). Análisis de la posesión del balón en el fútbol juvenil en relación a variables de situación: Estudio de caso. Cuadernos de Psicología del Deporte, 20(20), 128-138. https://doi.org/10.6018/cpd.370261

15. Fothergill, M., Wolfson, S., y Neave, N. (2017). Testosterone and cortisol responses in male soccer players: The effect of home and away venues. Physiology \& Behavior 177(1), 215-220. https://doi.org/10.1016/j.physbeh.2017.04.021

16. Furley, P., Schweizer, G., y Memmert, D. (2018). Thin Slices of Athletes' Nonverbal Behavior Give Away Game Location: Testing the Territoriality Hypothesis of the Home Game Advantage. Evolutionary psychology: an international journal of evolutionary approaches to psychology and behavior, 16(2), 1-12. https://doi.org/10.1177/1474704918776456

17. García-Calvo, T., Sánchez-Miguel, P. A., LeoMarcos, F. M., Martín-Clemente, E., y MendesLeal, J. A. (2011). Análisis de la ventaja de jugar en casa en el fútbol profesional español. Revista Alto Rendimiento, 38. http://altorendimiento.com/analisis-de-la-ventajade-jugar-en-casa-en-el-futbol-profesionalespanol/

18. Glamser, F. D. (1990). Contest location, player misconduct, and race: a case from english soccer. Journal of Sport Behavior 13(1), 41-49.

19. Gómez-Bantel, A. (2015). Football clubs as symbols of regional identities. Soccer \& Society,
17(5),

692-702.

https://doi.org/10.1080/14660970.2015.1100430

20. González-García, H., y Martínez-Martínez, F. D. (2019). Análisis de la influencia de los goles encajados en la clasificación final de las principales ligas de fútbol europeas. Journal of Sport and Health Research, 11(3), 305-314. http://www.journalshr.com/papers/Vol\%2011_N \%203/JSHR\%20V11_3_8.pdf

21. Goumas, C. (2014a). Tyranny of distance: Home advantage and travel in international club football. International Journal of Performance Analysis in Sport 14(1), 1-13. https://doi.org/10.1080/24748668.2014.1186869 8

22. Goumas, C. (2014b). Home advantage and referee bias in European football, European Journal of Sport Science, 14(sup1), 243-249. https://doi.org/10.1080/17461391.2012.686062

23. Guschwan, M. (2015). Performance in the stands. Soccer \& Society, 17(3), 290-316. https://doi.org/10.1080/14660970.2015.1082758

24. Hernández-Bonilla, L. D. (2019). Intervención psicológica en la selección absoluta de futsal de Costa Rica para el Mundial de Colombia 2016. Revista de Psicología Aplicada al Deporte y al Ejercicio Físico, 4(2), 1-9. https://www.revistapsicologiaaplicadadeporteyej ercicio.org/art/rpadef2019a16

25. Inan, T. (2020). The effect of crowd support on home-field advantage: evidence from european football. Annals of Applied Sport Science, 8(3), 19. https://doi.org/10.29252/aassjournal.806

26. Jacklin, J. P. (2005). Temporal changes in home advantage in English football since the Second World War: What explains improved away performance? Journal of Sports Sciences, 23(7), 669-679.

https://doi.org/10.1080/02640410400021948

27. Jiménez-Sánchez, Á., y Lavín, J. M. (2020). Home advantage in European soccer without 


\section{Jugar sin público y ventaja local}

crowd. Soccer \& Society, (Oct), 1-14. https://doi.org/10.1080/14660970.2020.1830067

28. Lago-Peñas, C., y Lago-Ballesteros, J. (2011). Game location and team quality effects on performance profiles in professional soccer. Journal of sports science \& medicine, 10(3), 465471. https://www.ncbi.nlm.nih.gov/pmc/articles/PMC 3737821/

29. Lehman, D. R., y Reifman, A. (1987). Spectator influence on basketball officiating. Journal of Social Psychology, 137(6), 673-675.

30. Loughead, T. M., Carron, A. V., Bray, S. R., y Kim, A. J. (2003). Facility familiarity and the home advantage in professional sports. International Journal of Sport and Exercise Psychology, 1(3), 264-274. https://doi.org/10.1080/1612197X.2003.9671718

31. McGuire, E. J., Courneya, K. S., Widmeyer, W. N., y Carron, A. V. (1992). Aggression as a potential mediator of the home advantage in professional ice hockey. Journal of Sport \& Exercise Psychology, 14(2), 148-158. https://doi.org/10.1123/jsep.14.2.148

32. Myers, T. D. (2014). Achieving external validity in home advantage research: generalizing crowd noise effects. Front. Psychol., 5(532), 1-7. https://doi.org/10.3389/fpsyg.2014.00532

33. Neave, N., y Wolfson, S. (2003). Testosterone, territoriality, and the 'home advantage'. Physiology \& Behavior 78(2), 269-275. https://doi.org/https://doi.org/10.1016/S00319384(02)00969-1

34. Nevill, A. M., Balmer, N. J. y Williams, A.M. (1999). Crowd influence on decisions in association football. The Lancet, 353(9162), 1416. https://doi.org/10.1016/S0140-6736(99)01299-4

35.Nevill, A. M., Newell, S. M., y Gale, S. (1996). Factors associated with home advantage in English and Scottish soccer matches. Journal of Sports Sciences, $\quad 14(2), \quad 181-186$. https://doi.org/10.1080/02640419608727700
36. Olmedilla-Zafra, A. (2012). Manual de psicología de la actividad física y del deporte. Murcia: Diego Marín.

37.Page, P., y Page, K. (2007). The second leg home advantage: Evidence from European football cup competitions. Journal of Sports Sciences, 25(14), 1547-1556.

https://doi.org/10.1080/02640410701275219

38.Picazo-Tadeo, A. J., González-Gómez, F., y Guardiola, J. (2017). Does the crowd matter in refereeing decisions? Evidence from Spanish soccer. International Journal of Sport and Exercise Psychology, 15(5), 447-459. https://doi.org/10.1080/1612197X.2015.1126852

39.Pollard, R. (2007). Worldwide regional variations in home advantage in association football. Journal of Sports Sciencies, 24(3), 231-240. https://doi.org/10.1080/02640410500141836

40.Pollard, R. (2008). Home advantage in soccer: A retrospective analysis. Journal of Sports Sciences $4(3)$, 237-248. https://doi.org/10.1080/02640418608732122

41.Pollard, R., y Gómez, M. A. (2009). Home advantage in football in South-West Europe: Longterm trends, regional variation, and team differences. European Journal of Sport Science, 9(6), 341-352. https://doi.org/10.1080/17461390903009133

42.Pollard, R., y Gómez, M. A. (2012). Comparison of home advantage in men's and women's football leagues in Europe. European Journal of Sport Science, $\quad$ 14(sup1), 77-83. https://doi.org/10.1080/17461391.2011.651490

43.Pollard, R., y Gómez, M. A. (2014). Components of home advantage in 157 national soccer leagues worldwide. International Journal of Sport and Exercise Psychology, 12(3), 218-233. https://doi.org/10.1080/1612197X.2014.888245

44.Rodríguez-Quijada, M. (2015). ¿Existe la ventaja de jugar en casa? Mundo Entrenamiento. 


\section{Jiménez-Sánchez et al.}

https://mundoentrenamiento.com/existe-laventaja-jugar-casa/

45. Saavedra-García, M., Gutiérrez-Aguilar, O., Fernández-Romero, J. J., y Sa-Marques, P. (2015). Measuring home advantage in spanish football (1928-2011). Revista Internacional de Medicina y Ciencias de la Actividad Física y el Deporte, 15(57), 181-194. http://cdeporte.rediris.es/revista/revista57/artventa ja550e.pdf

46. Sánchez, P. A., García-Calvo, T., Leo, F. M., Pollard, R., y Gómez, M. A. (2009). An Analysis of Home Advantage in the Top Two Spanish Professional Football Leagues. Perceptual and Motor Skills, 108(3), 789-797. https://doi.org/https://doi.org/10.2466/pms.108.3.7 89-797

47.Schwartz, B., y Barsky, S. F. (1977). The Home Advantage. Social Forces, 55(3), 641-661, https://doi.org/10.1093/sf/55.3.641

48. Seçkin, A. (2006). Home Advantage in Association Football: Evidence from Turkish Super League. Proceedings of International Conference on Policy Modeling (ECOMOD, 2006). Hong Kong, June 2830. https://ecomod.net/sites/default/files/documentconference/ecomod2006/1596.pdf

49. Simonenkova, I., y Sopov, V. (2020). Psychological Skills of Highly Qualified Football Players, Determining the Effectiveness of their Playing Activities. Revista De Psicología Del Deporte, 29(2), 171-175. https://www.rpdonline.com/index.php/rpd/article/view/13

50. Triplett, N. (1898). The Dynamogenic Factors in Pacemaking and Competition. The American Journal of Psychology, 9(4), 507-533. https://doi.org/10.2307/1412188

51.Tucker, W., Mellalieu, D. S., James, N., y Taylor, B. J. (2005). Game Location Effects in Professional Soccer: A Case Study. International Journal of Performance Analysis in Sport, 5(2), 23-35. https://doi.org/10.1080/24748668.2005.11868325
52.Unkelbach, C., y Memmert, D. (2010). Crowd Noise as a Cue in Referee Decisions Contributes to the Home Advantage. Journal of Sport and Exercise Psychology, 32(4), 483-498. https://doi.org/10.1123/jsep.32.4.483

53.Varca, P. E. (1980). An Analysis of Home and Away Game Performance of Male College Basketball Teams. Journal of Sport Psychology, 2(3), 245-257. https://doi.org/10.1123/jsp.2.3.245

54.Wright, E. F., Voyer, D., Wright, R. D., y Roney, C. (1995). Supporting audience and performance under pressure: The home-ice disadvantage in hockey championships. Journal of sport behavior, 18(1), 21-28.

55.Zajonc, R. B. (1965). Social facilitation. Science, 149(3681), 269-274. https://doi.org/10.1126/science.149.3681.269

\section{ANEXOS}

Correlaciones de Pearson entre variables según el país: https://drive.google.com/file/d/1w8nyvJB08KcFOehr mtPjRI1I9-rUNGYd/view?usp=sharing 